\title{
Gastric enzymes as a screening test for gastric cancer
}

\author{
P J FINCH, F P RYAN, K ROGERS, AND S HOLT \\ From the Departments of Gastroenterology and Histopathology, Northern General Hospital, Herries Road, \\ Sheffield
}

SUMMARY Levels of lactic dehydrogenase and beta-glucuronidase were measured in gastric wash samples obtained from 445 patients over 40 years old attending a routine diagnostic endoscopy clinic. An index was derived from the two levels and used as a test for the presence of gastric cancer. Of the 24 patients with an endoscopic diagnosis of gastric cancer, $21(91 \cdot 3 \%)$ proved positive on this test, including all four cases of early gastric cancer, which were found at repeat endoscopy. The specificity of the test for gastric cancer was $81 \cdot 3 \%$. Among those patients with false positive results who had endoscopic biopsy were four out of the five cases of severe dysplasia, and four out of the 13 cases of type $2 \mathrm{~B}$ intestinal metaplasia. Atrophic gastritis alone or with intestinal metaplasia was found in $95.9 \%$ of the false positives who were biopsied.

Gastric cancer, the fourth most common malignancy in this country, has an appalling prognosis $(4.7 \%$ five year survival in the $\left.\mathrm{UK}^{\prime}\right)$. Earlier diagnosis at the stage of 'early gastric cancer' (carcinoma in which the depth of invasion is limited to the submucosal layer of the stomach on histological examination') has a much improved survival (70).4\% at five years'). This has led to considerable interest in detecting early gastric cancer and any possible 'premalignant' gastric mucosal changes such as severe dysplasia and type 2B intestinal metaplasia (IM). Some means of improving the detection rate at routine endoscopy is thus desirable.

Confirming earlier studies, ${ }^{3+}$ a recent report showed that the levels of the enzymes lactic dehydrogenase and beta-glucuronidase in gastric juice were raised in patients with gastric cancer, including early gastric cancer. The study population was, however, a highly selected group of patients before surgery with a high proportion of cancer cases (42 out of 113 tested).

We have extended our study to an unselected group consisting of those patients presenting for routine diagnostic upper gastrointestinal endoscopy over a period of two years. The aim of the study was to assess if measurement of intragastric lactic

Address for correspondence: Dr P J Finch. Department of Gastroenterology. Royal I.iverpool Hospital. Prescot Street. Liverpeol

Received for publication 27 Junc 1986. dehydrogenase and beta-glucuronidase might prove a useful adjunct to upper gastrointestinal endoscopy in detecting both early cancers and premalignant mucosal changes.

\section{Methods}

PATIENTS

The study population consisted of a prospective group of all patients over the age of 40 years presenting for routine diagnostic upper gastrointestinal endoscopy to our clinic. The presence of blood or food in the stomach was the only exclusion.

\section{ENDOSCOPY}

During the endoscopy, resting juice was aspirated and discarded. Before entering the duodenum (to minimise duodenogastric reflux), $100 \mathrm{ml}$ Sorensen's buffer (67 mmol phosphate $\mathrm{pH} 7.5)$ containing $4 \mathrm{~g} / \mathrm{l}$ xylose, warmed to body temperature, was sprayed via the biopsy channel of the endoscope upon the antral mucosa. The buffer ran down over the mucosa, washing it, bearing away the adherent juice and pooling in the fundus, where it was collected by aspiration during retroversion of the endoscope. This procedure added only a short time to the duration of the endoscopy.

Patients were grouped according to the endoscopic diagnosis to enable between group comparisons of enzyme level. The groupings used were: (1) Normal 
endoscopy, (2) Oesophagitis or oesophageal ulceration, (3) Duodenitis or duodenal ulceration, (4) Acute erosive gastritis (usually NSAI related), (5) Chronic gastritis, (6) Gastric ulceration, (7) Isolated gastric polyp, (8) Gastric carcinoma.

Chronic gastritis (5) was recognised by erythema, granularity, superficial ulceration or haemorrhage, chronic erosions, mucosal atrophy, visible areae gastricae or intestinal metaplasia.

\section{HISTOLOGY}

An average of three biopsies were taken of any suspicious lesion or area of gastritis and examined by routine histology. Patients were also grouped on the basis of the worst histological feature present. The groupings were: (1) No biopsy, (2) Normal histology, (3) Superficial gastritis, (4) Atrophic gastritis, (5) Gastritis with intestinal metaplasia, Adenocarcinoma.

The 'no biopsy' group either had endoscopically normal stomachs or mild gastritis and other pathology to explain their symptoms, hence no biopsy was taken.

Histological sections from 83 consecutive patients without carcinoma undergoing biopsy were also examined after staining by the high-iron diamine/ Alcian blue method' for the presence of sialomucin and sulphomucin containing intestinal metaplasia.

\section{ENZYMES}

The gastric washings were analysed for lactic dehydrogenase (after the method of Henry' adapted for multiple analysis) and for beta-glucuronidase (Sigma kit 325), within one week, having been stored in small aliquots at $-60^{\circ} \mathrm{C}$.
STATISTICAL ANALYSIS

One way analysis of variance was used to assess between group differences in enzyme level utilising data transformed to the cubed root to normalise the distribution.

\section{Results}

There were 30 cases of obvious advanced gastric cancer which were excluded because of the presence of large amounts of blood or food in the stomach. The mean age of the 445 patients ( 215 men) who were tested was 67.1 years (SD 11.6). The levels of both lactic dehydrogenase and beta-glucuronidase showed a marked positive skew in their distributions. In order to normalise distributions and also to make results comparable with those in the earlier study, the sum of the cubed root of the lactic dehydrogenase level in IU/l, and the cubed root of the betaglucuronidase level in $\mathrm{IU} / \mathrm{ml}$, was calculated to form an index which could be used as a test for gastric cancer. The mean index for patients with endoscopically normal stomachs $(n=131)$ was $3.4($ SD $1 \cdot 6)$. A level of 5.8 and greater was taken as positive, this figure representing $93 \%$ of a normal distribution (mean plus 1.5 times standard deviation) based on those patients with endoscopically normal stomachs (normal endoscopy, oesophageal or duodenal pathology).

\section{ENDOSCOPIC DIAGNOSIS}

The number of patients, and the positive test results that is, an index greater than or equal to $5 \cdot 8$, for each endoscopic group are seen in Table 1. Of the 24 carcinoma cases, $21(87.5 \%)$ were positive but one of

Table 1 Endoscopic diagnosis

\begin{tabular}{|c|c|c|c|c|c|c|c|c|}
\hline & NO & $O E$ & $D U$ & $A G$ & $C G$ & $G U$ & $P O$ & $C A$ \\
\hline No. & 62 & 31 & 38 & 12 & 190 & 81 & 7 & $24^{*}$ \\
\hline Age & $64 \cdot 0$ & $64 \cdot 1$ & $63 \cdot 3$ & $71 \cdot 1$ & $67 \cdot 8$ & $67 \cdot 6$ & $78 \cdot 1+$ & $71 \cdot 8+$ \\
\hline SD & $13 \cdot 5$ & $10) \cdot 8$ & $10 \cdot 1$ & $9 \cdot 5$ & $11 \cdot 5$ & $10 \cdot 9$ & $7 \cdot 4$ & $10 \cdot 1$ \\
\hline Positive & 8 & 1 & 1 & () & 48 & 18 & 3 & 21 \\
\hline \% pos. & $12 \cdot 9$ & $3 \cdot 2$ & $2 \cdot 6$ & 0 & $25 \cdot 3$ & $22 \cdot 2$ & 42.9 & $87 \cdot 5$ \\
\hline Index & 3.918 & $3 \cdot 246$ & $2 \cdot 692$ & $3 \cdot 107$ & $4 \cdot 412$ & $4 \cdot 261$ & $4 \cdot 711$ & 7.459 \\
\hline \multirow[t]{2}{*}{ SD } & 1.739 & 1.475 & 1.259 & $1 \cdot 414$ & $2 \cdot() 42$ & 1.698 & $2 \cdot 152$ & $2 \cdot 351$ \\
\hline & $\mathrm{F}_{4,37}^{7} 15$ & & & & & & & \\
\hline \multicolumn{9}{|c|}{ Between group differences: } \\
\hline & & 1 & 1 & $p<0 \cdot 05$ & 1 & & & \\
\hline & & I & 1 & & $\bar{p}<(0.05$ & 1 & & \\
\hline & 1 & $p<0.05$ & 1 & & $p<0.05$ & & 1 & \\
\hline
\end{tabular}

Number of patients and mean age and SD, number with index $>=5.8$ and $\%$ of group positive, mean index and SD, and analysis of variance, for patients grouped according to endoscopic diagnosis. (NO normal; OE ocsophageal pathology; DU duodenal pathology; AG acute erosive gastritis: $\mathrm{CG}$ chronic gastritis; GU gastric ulcer; $\mathrm{PO}$ isolated polyp; $\mathrm{CA}$ gastric carcinoma.)

${ }^{*}$ Includes one case of pancreatic cancer mistakenly diagnosed as gastric cancer; $†$ Significantly different from the other groups $(p<0) \cdot(01)$. 
Table 2 Histological diagnosis

\begin{tabular}{|c|c|c|c|c|c|c|c|c|}
\hline &.$N B$ &.$N()$ & $S(;$ & $A(;$ & $M$ & $C A$ & $E C$ & $A C$ \\
\hline No. & 124 & 34 & 15 & 110 & $1+4$ & 18 & 4 & 14 \\
\hline Positive & 11 & 2 & 1 & 28 & 43 & 15 & 4 & 11 \\
\hline "o pos. & 8.9 & 5.9 & 6.6 & 25.5 & 29.9 & 83.3 & $1(x)$ & $7 x \cdot 6$ \\
\hline Mcan & 3.491 & 3.817 & 3.1135 & 4.276 & 4.690 & $7 \cdot 292$ & 0.154 & $7 \cdot 512$ \\
\hline SD & 1.683 & 1.484 & 1.365 & $2 \cdot(1) 27$ & 2.1888 & $2 \cdot 507$ & 1.367 & 2.459 \\
\hline
\end{tabular}

Betweengroup differences:

\begin{tabular}{|c|c|c|c|}
\hline erences: & $\perp$ & $\perp$ & $|\mathrm{p}<(1) \cdot(1)|$ \\
\hline 1 & & $p<0.105$ & 1 \\
\hline & $\mid \mathrm{p}<0.0 .5$ & 1 & \\
\hline
\end{tabular}

The number of patients. number with index $>=5.8$ and \% of group positive, mean index and standard deviation, and analysis of variance, for patients grouped according to histological diagnosis. (NB no biospy; NO normal: SG superficial gastritis; AG at rophic gastritis; IM gastritis with intestinal metaplasia: ( A adenocarcinoma: EC 'early gastric cancer: AC advanced gastric cancer.)

the negatives was later found to be suffering from a pancreatic carcinoma invading the gastric wall, and excluding this case the sensitivity rose to $91 \cdot 3 \%$. The normal stomach groups (NO, OE and DU) were rarely positive, and the gastritis and gastric ulcer groups had intermediate numbers of positives. As a test for gastric cancer, this index had a sensitivity of $91.3 \%$ and a specificity of $81.3 \%$. () the false positives. $87 \cdot 3 \%$ had gastritis ( $(\mathrm{C}, \mathrm{GU}$ and $\mathrm{PO})$.

The carcinoma group included four calses of early gastric cancer, and one case of linitus plastica, all of which were positive. All four cases of early gastric cancer were found at subsequent endoscopy, which was repeated in three because of positive enzymes. suspicious histology and a high degree of clinical suspicion. while in the other the cancer was found during follow up for an adenomatous polyp. Thus four of the "false positives' were subsequently shown to have cancer. Although advanced cancer cases tended to have the higher enzyme concentrations, occasional cases were negative, and no relationship between the index and he staging of the tumour could reliably be identified.

Also seen in Table 1 are the mean index and standard deviation for each group with andysis of variance. The carcinoma group had a significantly higher index than all other groups. Patients with gastric ulcer had a significantly higher index than those with oesophageal and duodenal pathology, and also those with acute gastritis. as did the chronic gastritis group. Patients with duodenal pathology had a significantly lower index than all other groups except those with oesophageal pathology or acute erosive gastritis.

HISTOIOCGICAI DIACRNOSIS

The number of patients, the positive test results-that is, an index greater than or equal to 5.8 and percentage positive for each histological group are seen in Table 2. Six of the 24 carcinoma cases showed necrotic slough only, on biopsy and are not included in the carcinoma group. Only a few of the no biopsy, normal and superficial gastritis groups were positive. a high proportion of the carcinoma group were positive. while the atrophic gastritis and intestinal metaplasia groups had an intermediate number of positives. Four out of five calses of severe dysplasia were positive. Based on the histology - that is. excluding the no biopsy group, the sensitivity as a test for carcinoma was $83.3 \%$ while the specificity was $75.6 \%$. Atrophic gastritis alone or with intestinal metaplasia was found in $95.9 \%$ of the false positives.

The mean index and standard deviation for each group are shown graphically in the Figure and also in

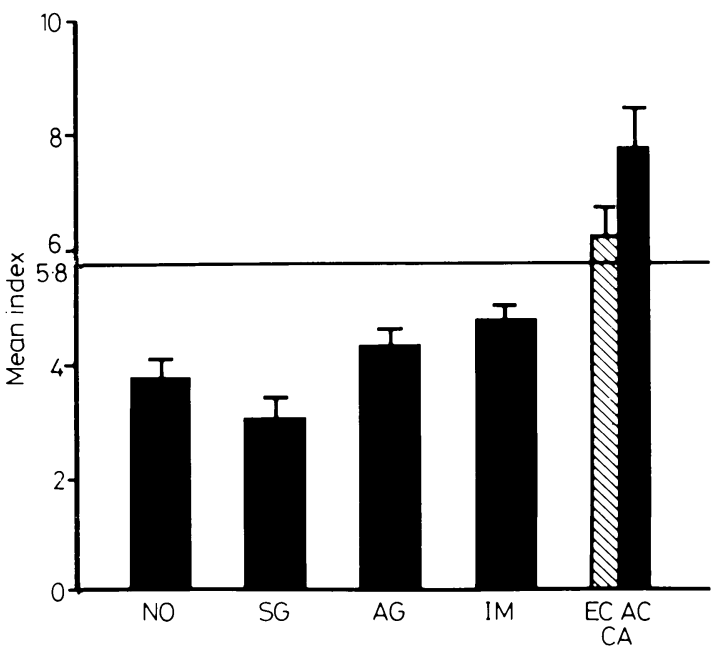

Fig. Mean index with standard error bars for patients grouped according to histological diagnosis 
Table 2 with analysis of variance. The mean index in the carcinoma group was significantly higher than all other groups. The mean index in the intestinal metaplasia group was significantly higher than that in the no biopsy, normal and superficial gastritis groups. The mean index in the atrophic gastritis group was significantly higher than that in the no biopsy and superficial gastritis groups.

\section{MUCIN HISTOCHEMISTRY}

Biopsies from 83 consecutive patients were classified according to the mucin histochemistry. The histological findings on these biopsies were normal (nine), superficial gastritis (12), chronic gastritis (62) and intestinal metaplasia. Most cases of sulphomucin bearing intestinal metaplasia also had sialomucin but were grouped separately. Forty one sections showed neither sialomucin nor sulphomucin intestinal metaplasia and of these, seven cases $(17 \%)$ were associated with a positive index. Twenty nine sections showed only sialomucin intestinal metaplasia and of these, three cases $(10 \cdot 3 \%)$ had positive enzymes. Thirteen sections showed sulphomucin intestinal metaplasia and of these, four cases $(30 \cdot 8 \%)$ had positive enzymes.

\section{Discussion}

Gastric wash enzymes were used as a screening test for gastric cancer in a general endoscopy population. The test is quick to do and does not add further to the inconvenience suffered by the patient from the endoscopy. Furthermore the enzyme assay method is simple, cheap, and already available in many laboratories, unlike other proposed markers of gastric malignancy such as foetal sulphoglycoprotein antigen (FSA). ${ }^{\text {" }}$ Unfortunately there are no satisfactory serum markers that could be used in screening for gastric cancer.

Not all cases of gastric cancer were positive and the sensitivity of the test was $91 \cdot 3 \%$. All four cases of early gastric cancer, and one case of linitus plastica, however, were positive. While the specificity of the test $(81.3 \%)$ was low, this has to be viewed in terms of screening rather than diagnosis. The predictive power of the test $(72 \%)$ is higher than that of FSA $(70 \%) .{ }^{\circ}$ The possibility that an early gastric cancer was missed may account for some of the false positives. Certainly four of our patients who had positive enzymes were subjected to repeat endoscopy and were subsequently found to have recognisable early gastric cancer.

We have shown that $95.9 \%$ of the false positives had some degree of atrophic gastritis with or without intestinal metaplasia. There is growing acceptance of the concept of intestinal metaplasia, especially the sulphomucin variant, as a premalignant lesion:" four of the 13 cases in our study with sulphomucin intestinal metaplasia were positive on this test. A more important premalignant lesion in the stomach is severe dysplasia," and four of the five cases with severe dysplasia in our study were positive. An earlier study" found severe dysplasia in three out of 17 false positives, but not at all in 17 matched negatives. In the same study, sulphomucin intestinal metaplasia was found in eight of 17 false positives but only one of 17 matched negatives.

These findings suggest that the false positive group may be at increased risk of developing gastric cancer in the future. We propose to follow up this false positive group with periodic endoscopic surveillance, together with a matched group of negatives, when the true specificity of this test may increase.

In conclusion, the measurement of the enzymes lactic dehydrogenase and beta-glucuronidase in gastric wash samples could be a useful screening test for early gastric cancer in an endoscopy population.

\section{References}

1 Fielding JL, Ellis DJ, Jones BG, et al. Natural history of early gastric cancer: results of a 10 year regional survey. Br Med J 1980; 281 : 965-7.

2 Murakami T. Early cancer of stomach. World J Surg 1979; 3: 685-91.

3 Piper DW, Griffith EM, Irving LG, Fenton BH. Value of bGlucuronidase in gastric juice in the diagnosis of gastric cancer. Gastroenterology 1966; 51: 172-9.

4 Smyrniotis F, Shenker S, O’Donnell J, Schiff L. Lactic Dehydrogenase activity in gastric juice for the diagnosis of gastric cancer. Am J Dig Dis 1962; 7: 712-6.

5 Rogers K, Roberts GM, Williams GT. Gastric juice enzymes - an aid to diagnosis of gastric cancer? Lancet 1981; 1: 1124-5.

6 Spicer SS. Diamine methods for differentiating mucosubstances histochemically. J Histochem Cytochem 1965; 13: 211-34.

7 Henry RJ. Clinical chemistry - Principles and techniques. London: Harper and Row, 1974.

8 Hakkinen I. Gastric fetal sulfoglycoprotein antigen. Antibiotics Chemother 1978; 22: 132-40.

9 Jass JR. Filipe MI. A variant of intestinal metaplasia associated with gastric carcinoma: a histochemical study. Histopathology 1979; 3: 191-9.

10 Morson BC, Sobin LH, Grundmann E, Johansen A, Nagayo T, Serck-Hanssen A. Precancerous conditions and epithelial dysplasia in the stomach. J Clin Pathol 1980; 33: 711-21.

11 Williams GT, Rogers K. Elevated gastric juice enzymes - a marker for increased gastric cancer risk. Clin Oncol 1984; 10: 319-23. 